\title{
THE HOMOTOPY THEORY OF PROJECTIVE MODULES
}

BY H. BASS AND S. SCHANUEL

Communicated by Raoul Bott, February 13, 1962

Serre $[8 ; 9]$ has established the rudiments of a dictionary for translating the language of projective modules into that of vector bundles. With this point of departure we have attempted to adapt some of the results and methods of homotopy theory to certain purely arithmetic and even noncommutative settings. Detailed proofs of our results will appear elsewhere. The authors are very grateful to Albrecht Dold who has educated them on the relevant parts of bundle theory.

We first recall briefly the topological setting which our theorems parody. $[X, Y]$ denotes the homotopy classes of maps of $X$ to $Y$. If $f: A \rightarrow B$ induces homotopy isomorphisms in dimensions $\leqq r$ and if $X$ is a finite $\mathrm{CW}$ complex of dimension $\leqq m$, then one shows easily (see $[10$, Appendix $]$ ) that $[X, A] \rightarrow[X, B]$ is surjective for $r \geqq m$ and injective for $r>m$. As special cases we have

I. $\left[X, B_{O(r)}\right] \rightarrow\left[X, B_{O(r+1)}\right]$ is surjective for $r \geqq m$ and injective for $r>m$; and

II. $[X, O(r)] \rightarrow[X, O(r+1)]$ is surjective for $r \geqq m+1$ and injective for $r>m+1$.

Here $B_{O(r)}$ is the classifying space of the orthogonal group $O(r)$. The classification theorem $[11, \S 19]$ says that $\left[X, B_{O(r)}\right]$ represents the functor, [(equivalence classes of) real $r$-plane bundles over $X$ ], and then the map in $I$ is obtained by adding a trivial line bundle. Moreover, the argument in $[11, \S 18]$ shows that $[X, O(r)] / \pi_{0}(O(r))$ represents the functor [real $r$-plane bundles over $S X$ ], where $S X$ is the (reduced) suspension of $X$.

Atiyah and Hirzebruch [1] define a functor $K^{*}(X)=K^{0}(X) \oplus K^{1}(X)$ as follows: $K^{0}(X)$ is the Grothendieck ring of vector bundles over $X$. Choosing a base point gives $K^{0}$ an augmentation, and $K^{1}(X)$ is then the augmentation ideal of $K^{0}(S X)$. Thus I and II above define a kind of stable range for $K^{0}$ and $K^{1}$ respectively.

Now let $R$ be a commutative ring, $X$ its spectrum of maximal ideals, and $\Lambda$ an $R$-algebra finitely generated as an $R$-module. We require only that $X$ be what might be called a "Zariski complex," i.e. that each closed set be a finite union of irreducible closed sets (see [4, $\S 3])$. If $P$ is a $\Lambda$-module and $x \in X$ then $P_{x}$ is the localization of $P$ at $x$; we say $f$-rank $P \geqq r$ if $P_{x}$ contains a $\Lambda_{x}$-free direct summand of rank $r$ for all $x$. Note that $P$ is required to be neither projective nor 
finitely generated. The following two theorems are a strong translation of I above.

Theorem 1 (Serre, see [8] or [4]). If f-rank $P>\operatorname{dim} X$ then $P \cong \Lambda \oplus P^{\prime}$ for some $P^{\prime}$.

Theorem 2. Suppose $P=P_{0} \oplus M$ with $P_{0}$ projective of $f$-rank $>\operatorname{dim} X$. Then if $Q$ is finitely generated and projective

$$
Q \oplus P \cong Q \oplus P^{\prime} \Rightarrow P \cong P^{\prime} \text {. }
$$

Observe that if $f$-rank $P>2 \operatorname{dim} X$ then by Theorem $1 P$ automatically has the form required for Theorem 2 .

Next we define $K^{*}(\Lambda)=K^{0}(\Lambda) \oplus K^{1}(\Lambda) . K^{0}(\Lambda)$ is the Grothendieck group of finitely generated projective $\Lambda$-modules. In defining $K^{1}$, as well as in proving Theorem 2, we use notions visibly borrowed from J. H. C. Whitehead's theory of simple homotopy types [12]. Let $\Lambda\langle t\rangle$ denote the group ring over $\Lambda$ of an infinite cyclic group generated by $t$. If $P$ and $Q$ are $\Lambda\langle t\rangle$-modules we call them "homotopic" if there is a $\Lambda$-isomorphism $f: P \rightarrow Q$ for which $f^{-1} t^{-1} f t$ is in the commutator subgroup of the $\Lambda$-automorphisms of $P$. This said, let $K^{*}(\Lambda)$ be the Grothendieck group generated by homotopy classes of $\Lambda\langle t\rangle$-modules which are finitely generated and projective as $\Lambda$-modules, modulo the relations given by short exact sequences. Disregarding the action of $t$ gives a (split) epimorphism $K^{*}(\Lambda) \rightarrow K^{0}(\Lambda)$ whose kernel we call $K^{1}(\Lambda)$.

Proposition. $K^{1}(\Lambda)$ is naturally isomorphic to the Whitehead group $G L(\Lambda) /[G L(\Lambda), G L(\Lambda)], G L(\Lambda)$ being the infinite general linear group.

If we let $E_{r}(\Lambda)$ denote the subgroup of $G L_{r}(\Lambda)$ generated by the elementary matrices and $E(\Lambda)=\lim _{\rightarrow} E_{r}(\Lambda) \subset \lim _{\rightarrow} G L_{r}(\Lambda)=G L(\Lambda)$, the elements of $E$ represent essentially the "simple homotopy equivalences" in Whitehead's theory, and he proves [12] that $E(\Lambda)$ $=[G L(\Lambda), G L(\Lambda)]$. Thus, our $K^{1}(\Lambda)$ is $G L(\Lambda)$ reduced modulo (simple) homotopic triviality. In this light Theorem 3 below is an (incomplete) transcription of II above.

One of the principal reasons for not taking the Whitehead group as the definition of $K^{1}(\Lambda)$ is that, for purposes of relating the $K$-theory to the Brauer group of $R[2]$ it is essential that $K$ depend only on the category of $\Lambda$-modules, and not specifically on $\Lambda$.

Theorem 3. Suppose $\operatorname{dim} X \leqq m$.

(a) For $r \geqq m+1, G L_{r}(\Lambda)$ is generated by $G L_{m+1}(\Lambda)$ and $E_{r}(\Lambda)$.

(b) If $r>m+1, E_{r}(\Lambda)$ is normal in $G L_{r}(\Lambda)$.

(c) If $r \geqq 2(m+1), E_{r}(\Lambda)=\left[G L_{r}(\Lambda), G L_{r}(\Lambda)\right]$. 
The inadequacy of this theorem amounts to the following

Problem. If $r>m+1$ then does

$$
G L_{r}(\Lambda) \cap\left[G L_{r+1}(\Lambda), G L_{r+1}(\Lambda)\right]=\left[G L_{r}(\Lambda), G L_{r}(\Lambda)\right] \text { ? }
$$

If $\Lambda$ is a division algebra then $m=0$ and the affirmative solution of essentially this problem, together with Theorem 3, constitutes Dieudonné's theory of noncommutative determinants [5].

Finally, if $f: \Lambda \rightarrow \Gamma$ is a ring homomorphism a relative $K^{0}(f)$ (corresponding to bundles over the mapping cone) can be defined by a procedure like that for $K^{1}$, and it fits into an exact sequence

$$
K^{1}(\Lambda) \rightarrow K^{1}(\Gamma) \rightarrow K^{0}(f) \rightarrow K^{0}(\Lambda) \rightarrow K^{0}(\Gamma) .
$$

There is, of course, a good deal more structure present, of which we mention that $K^{0}(R)$ is a filtered " $\lambda$-ring," and $K^{0}(\Lambda)$ is a filtered $K^{0}(R)$-module, with filtrations vanishing beyond $\operatorname{dim} X$.

Two basic problems in these matters are: (1) What are the projective $k\left[X_{1}, \cdots, X_{m}\right]$-modules ( $k$ a field)? (See $[8]$ or $[9$, p. 243]). (2) Determine $K^{*}(Z \pi), \pi$ a finite group, or, more generally, $K^{*}(\Lambda)$ with $\Lambda$ an order in a semi-simple algebra over $Q$. When $\Lambda$ is a maximal order in a simple algebra some work of Eichler [6] permits one to describe $K^{0}(\Lambda)$ entirely in terms of the center of the algebra. In this number theoretic context our point of view suggests that the "units" $\left(K^{1}\right)$ are a kind of "suspension" of the functor "class group" $\left(K^{0}\right)$. Our results quickly yield the following mild contributions to the above problems.

THEOREM 4. If $k$ is a semi-local principal ideal domain and $R=k\left[X_{1}, \cdots, X_{m}\right]$ then a projective $R$-module $P$ of rank $r$ is free unless $1<r \leqq m$, in which case $P \oplus R^{m+1-r}$ is free.

THEOREM 5. Suppose $\Lambda$ is free of finite rank over $Z$.

(a) $K^{*}(\Lambda)$ is a finitely generated abelian group.

(b) $\left[G L_{r}(\Lambda), G L_{r}(\Lambda)\right]$ is finitely generated for $r \geqq 4$.

Theorem 1 and an argument of Kaplansky [7] suggested also the following transfinite analogue of Theorem 4, which is proved in [3].

Theorem 6. If $R$ is commutative Noetherian and $\operatorname{spec}(R)$ is connected then a projective $R$-module is either free or finitely generated.

\section{BiBLIOGRAPHY}

1. M. F. Atiyah and F. Hirzebruch, Vector bundles and homogeneous spaces, Proc. Sympos. Pure Math. Vol. 3, pp. 7-38, Amer. Math. Soc., Providence, R. I., 1961.

2. M. Auslander and O. Goldman, The Brauer group of a commutative ring, Trans. Amer. Math. Soc. 97 (1960), 367-409. 
3. H. Bass, Big projective modules are free (in preparation).

4. - Projective modules over algebras, Ann. of Math. 73 (1961), 532-542.

5. J. Dieudonné, Les déterminants sur un corps non commutatif, Bull. Soc. Math. France 71 (1943), 27-45.

6. M. Eichler, Über die Idealklassenzahl hypercomplexen Systeme, Math. Z. 43 (1938), 481-494.

7. I. Kaplansky, Modules over Dedekind rings and valuation rings, Trans. Amer. Math. Soc. 72 (1952), 327-340.

8. J.-P. Serre, Modules projectifs et espaces fibrés a fibre vectorielle, Séminaire P. Dubreil, exposé 23, Secrétariat mathématique, Paris, 1958.

9. - Faisceaux algebriques coherents, Ann. of Math. 61 (1955), 197-278.

10. E. Spanier, Infinite symmetric products, function spaces, and duality, Ann. of Math. 69 (1959), 142-198.

11. N. Steenrod, The topology of fiber bundles, Princeton Univ. Press, Princeton, N. J., 1951.

12. J. H. C. Whitehead, Simple homotopy types, Amer. J. Math. 72 (1950), 1-57.

Columbia University

\section{ACKNOWLEDGMENT}

BY H. D. BLOCK AND W. H. J. FUCHS

The result announced by H. D. Block and W. H. J. Fuchs (Research Announcements, Bull. Amer. Math. Soc. 67 (1961), 425) has been known for some time. We are indebted to Professor S. H. Crandall for the reference to N. Kryloff and N. Bogoliubov, Bull. Acad. Sci. URSS, Classe Phys. Math. Leningrad (1929), p. 471. 